\title{
Nicotinamide, a vitamin B3 ameliorates depressive behaviors independent of SIRT1 activity in mice
}

\author{
Zhuxi Liu, Caiqin Li, Xuelian Fan, Yifang Kuang, Xu Zhang, Lei Chen, Jinjing Song, Ying Zhou, Eiki Takahashi, \\ Guang He and Weidong Li* (i)
}

\begin{abstract}
Sirtuin 1 (SIRT1), is a nicotinamide adenine dinucleotide $\left(N A D^{+}\right)$-dependent protein deacetylase and a candidate gene for depression. Nicotinamide (NAM), a form of vitamin B3, is reported as a potential inhibitor of SIRT1. Our previous study found that the 24-h-restraint stress could induce long-term depressive-like phenotypes in mice. These mice displayed increased SIRT1 activity. Here, we studied whether NAM was capable of attenuating depressive behaviors through inhibiting SIRT1 activity. Surprisingly, the application of NAM significantly reversed the depressive behaviors but increased SIRT1 activity further. In contrast, the level of adenosine triphosphate (ATP) was reduced in the restraint model for depression, and recovered by the administration of NAM. Furthermore, the Sirt $7^{\text {flox }}$ flox; Nestin-Cre mice exhibited antidepressant behaviors and increased ATP levels. These data suggest that ATP plays an important role in depression pathogenesis, and NAM could be a potential treatment method for depression by regulating ATP independent of SIRT1 activity.
\end{abstract}

Keywords: SIRT1, Nicotinamide, ATP, Restraint animal model, Depression

\section{Introduction}

Depression is a common mental disorder accompanied by several psychological and emotional symptoms, and it affects approximately $4.4 \%$ of the world's population [1]; however, the complex mechanisms underlying the pathogenesis of depression remain unclearly. Sirtuin 1 (SIRT1) is a protein deacetylase that contribute to cellular regulation in vivo. Studies have suggested it is associated with depression [2-4]. However, whether its increase or decrease contributing to depressive phenotypes is controversial in animal modeling studies. Abe-Higuchi et al. reported that SIRT1 activity in the dentate gyrus (DG)

*Correspondence: liwd@sjtu.edu.cn

Bio-X Institutes, Key Laboratory for the Genetics of Development and Neuropsychiatric Disorders (Ministry of Education), Shanghai Key Laboratory of Psychotic Disorders, and Brain Science and Technology Research Center, Institute of Psychology and Behavioral Sciences,

Shanghai Jiao Tong University, Shanghai 200240, China of the hippocampus was reduced by chronic stress in mice [5], where Ferland et al. demonstrated that chronic stress exposure enhances SIRT1 activity in the DG of rats [6]. Kim et al. demonstrated that stress induced SIRT1 expression in the nucleus accumbens (NAc) and altering SIRT1 activity could regulate anxiety- and depressive-like behaviors [7]. Besides, SIRT1 overexpress mice were more susceptible to depression compared to their wildtype littermates [8]. According to our previous study, we constructed the 24-h-restraint model with long lasting depressive-like phenotypes [9]. In this model of depression, we found that SIRT1 activity was increased.

Nicotinamide (NAM), a form of Vitamin B3, has been suggested to be therapeutically effective against many diseases and conditions, and it is mainly applied to pellagra in the clinics. Evidence has also suggested that NAM aids recovery from depression or bipolar disorders $[10,11]$. Song et al. considered NAM to primarily work through original author(s) and the source, provide a link to the Creative Commons licence, and indicate if changes were made. The images or other third party material in this article are included in the article's Creative Commons licence, unless indicated otherwise in a credit line to the material. If material is not included in the article's Creative Commons licence and your intended use is not permitted by statutory regulation or exceeds the permitted use, you will need to obtain permission directly from the copyright holder. To view a copy of this licence, visit http://creativecommons.org/licenses/by/4.0/. The Creative Commons Public Domain Dedication waiver (http://creativeco mmons.org/publicdomain/zero/1.0/) applies to the data made available in this article, unless otherwise stated in a credit line to the data. 
increasing and decreasing monoamine-neurotransmitter synthesis and degradation respectively; they also considered NAM to potentially ameliorate depression through an antioxidative effect along with an increasing supply of nicotinamide adenine dinucleotide $\left(\mathrm{NAD}^{+}\right)$[12]. According to previous reports, NAM, which is produced by sirtuin enzymes, can inhibit the deacetylation of SIRT1 by binding to a conserved pocket adjacent to $\mathrm{NAD}^{+}$, thereby altering the $\mathrm{NAD}^{+}$co-substrate specificity of a Sir2 enzyme $[13,14]$.

Then, we investigated whether NAM could attenuate depressive behaviors through inhibiting SIRT1 activity in 24-h-restraint mouse model. Strikingly, our result showed that SIRT1 activity was further increased after the administration of NAM, along with the fully rescue of depressive behaviors in mice.

\section{Methods}

\section{Animals}

Adult male C57BL/6 mice (age 12 weeks old) were obtained from Beijing Vital River Laboratory Animal Technology Co., Ltd, which were housed in groups per cage in a temperature-controlled room with a standard 12-h light/12-h dark cycle (light on from 7:00 a.m. to 7:00 p.m., $\left.22{ }^{\circ} \mathrm{C} \pm 2{ }^{\circ} \mathrm{C}\right)$. We generated conditional Sirt1-

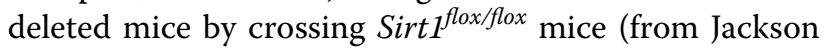
Lab, 008041) with Nestin-Cre mice. The Sirt $1^{f l o x f f l o x}$ mice were in a mixed 129SvJ/C57B6 background, while the Nestin-Cre mice were C57BL/6. The resulting Sirt $1^{f l o x /+}$; Nestin-Cre mice were mated with Sirt $1^{\text {flox/flox }}$ mice to obtain conditional Sirt1-knockout mice (Sirt $1^{f^{f l o x} / f l o x}$; Nes-

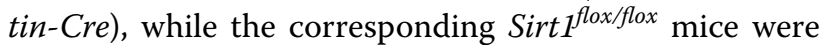
used as the control. All animal experiments were performed according to the guidelines approved by the University Committee on Animal Care and Use of Shanghai Jiao Tong University, China.

\section{Animal model of the $24-\mathrm{h}$ restraint}

The experimental procedure was performed according to the protocol described previously in our laboratory with slightly modifications [9]. The mice were placed in a ventilated clear plastic tube $(3 \mathrm{~cm}$ in diameter and $10 \mathrm{~cm}$ in length) and subjected to $24-\mathrm{h}$ restraint from 14:00 p.m. to 14:00 p.m. of the next day. Once the restraint ended, the mice were returned to their home cages with access to food and water ad libitum. The control group remained in their home cages without the 24-h-restraint procedure.

\section{Drug administration}

NAM reagent (Beyotime, Shanghai, China) was dissolved in drinking water at a $200 \mathrm{mg} / \mathrm{kg} /$ day dose $[15,16]$. NAM treatment lasted for 33 days, beginning 2 days after the end of the 24-h restraint, whereas control mice were provided with sufficient water. The solutions were changed every 3 days until the mice were sacrificed.

\section{Behavioral procedures}

After 5 weeks of 24-h restraint, behavioral tests were performed to verify the validity of the model to further study the mechanism of depression.

\section{Sucrose preference test (SPT)}

During the adaptation period, mice were individually housed to habituation with two bottles containing either $2 \%$ sucrose (Sigma-Aldrich) diluted in drinking water or drinking water alone. The habituation was sustained for 3 days with the positions of the two bottles exchanged every $24 \mathrm{~h}$. Water was removed at 4:00 p.m. for $17 \mathrm{~h}$, and the test was started at 10:00 a.m. the next day. The test was performed for a total of $24 \mathrm{~h}$, and then the positions of the two bottles were exchanged $12 \mathrm{~h}$ later.

\section{Forced swim test (FST)}

The FST, which measures acute stress responses, was performed during the light phase (10:00-17:00). Mice were placed in a $2 \mathrm{~L}$ beaker containing water $\left(24^{\circ} \mathrm{C} \pm 1{ }^{\circ} \mathrm{C}\right)$ for $5 \mathrm{~min}$ in a dim environment. The animals were analyzed for time spent on immobility.

\section{Western blot}

Hippocampus tissues were isolated and lysed in RIPA buffer supplemented with protease inhibitor cocktail (Roche). After centrifugation $(12,000 \mathrm{~g}$, for $20 \mathrm{~min}$ at $4{ }^{\circ} \mathrm{C}$ ), the supernatants were retained and quantified using a BCA protein assay kit (Beyotime, Shanghai, China). Equivalent proteins were subjected to $10 \%$ SDSPAGE and then transferred electrophoretically to polyvinylidene fluoride (PVDF) membranes at $300 \mathrm{~mA}$ for $60 \mathrm{~min}$. After blocking with 5\% bovine serum albumin (BSA), membranes were incubated with primary antibodies overnight at $4{ }^{\circ} \mathrm{C}$, namely Sirt 1 (1:1000, abcam) and $\beta$-actin (1:5000, CST). After incubation with appropriate horseradish peroxidase-conjugated secondary antibody (Millipore, USA), a high-sensitivity ECL reagent (Share-bio, China) was used. All the bands were analyzed with Image J.

\section{Quantitative real-time polymerase chain reaction}

Total RNA was extracted from the hippocampus using triazole (Invitrogen) reagent, and then real-time polymerase chain reaction (RT-PCR) amplification and sequencing were performed. The cDNA was synthesized using reagents from a reverse transcription kit (Takara) per manufacturer's instructions. Quantitative PCR 
(qPCR) was performed using an SYBR Green $5 \times$ PCR Master Mix (Takara) in an RT-PCR system performed on a Light Cycler 480 II (Roche). The primers are listed as follows: SIRT1-F: 5'-GCTGACGACTTCGACGAC G-3', SIRT1-R: 5'-TCGGTCAACAGGAGGTTGTCT3'; GAPDH-F: 5'-TGACGTGCCGCCTGGAGAAAC-3', GAPDH-R: 5'-CCGGCATCGAAGGTGGAAGAG-3'.

\section{SIRT1 activity assay}

To measure SIRT1 activity, the protein was extracted from tissue using nondenaturing lysates, and protein concentrations were measured with a BCA protein assay kit (Beyotime, China). SIRT1 activity was quantified with a SIRT1 fluorometric assay kit (Sigma, CS1040) per manufacturer instructions. In brief, the reaction was conducted at $37^{\circ} \mathrm{C}$ for $30 \mathrm{~min}$, and deacetylase activity was detected and measured using a multimode reader (Tecan Infinite Pro, Switzerland; excitation wavelength $=360 \mathrm{~nm}$, emission wavelength $=450 \mathrm{~nm}$ ) [17].

\section{ATP assay}

ATP levels of the hippocampus in mice were measured using a firefly luciferase-based ATP assay kit (Beyotime, Shanghai, China) per manufacturer instructions. Briefly, the tissue was lysed completely and centrifuged at $12,000 \mathrm{~g}$ for $5 \mathrm{~min}$ at $4{ }^{\circ} \mathrm{C}$. After the background ATP had been consumed by $100 \mu \mathrm{L}$ ATP detection working solution in a black 96-well plate, $20 \mu \mathrm{L}$ of each supernatant were added to each well and assayed by a multimode reader (Tecan Infinite Pro, Switzerland).

\section{Statistical analysis}

For all experiments, data were presented in terms of the mean \pm standard error of the mean (SEM) and analyzed using GraphPad Prism software. An unpaired t test was used to determine the statistical differences between the two groups, and one-way analysis of variance (ANOVA) was used to analyze the variance for three groups. Given a significant effect of the one-way ANOVA, further multiple comparisons were conducted with Turkey-Kramer
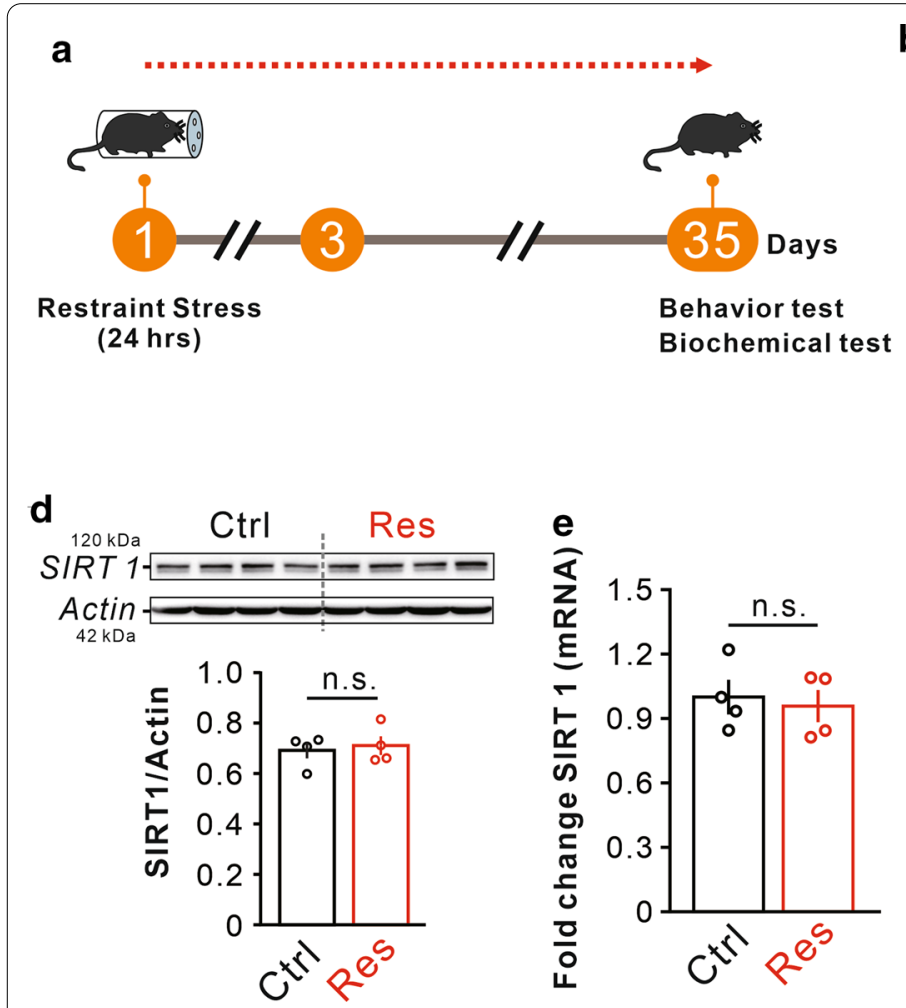
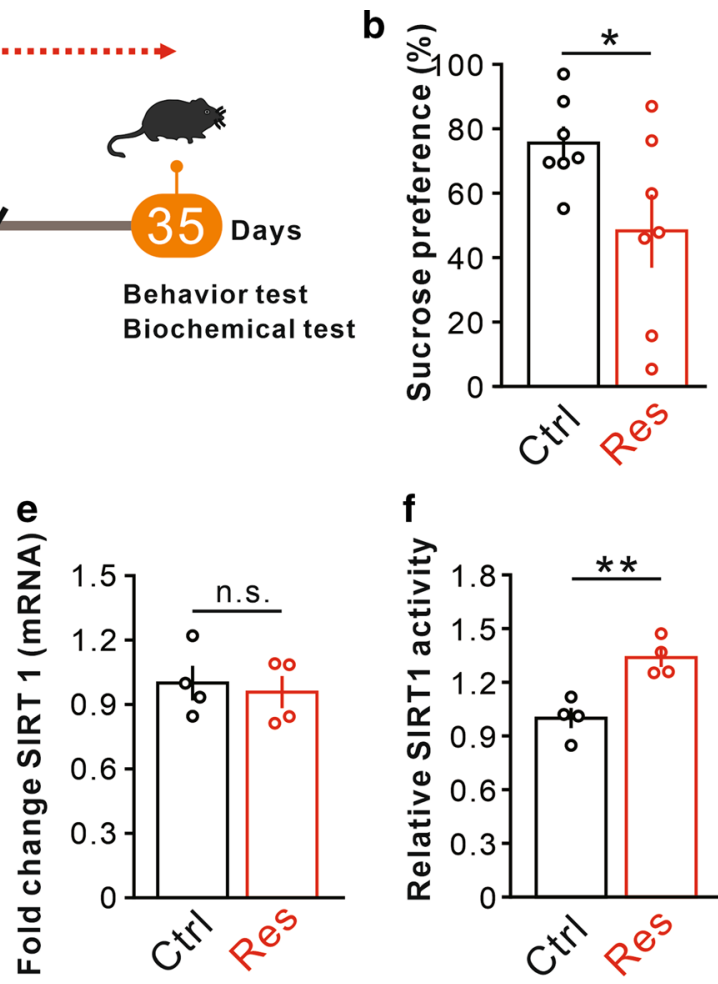

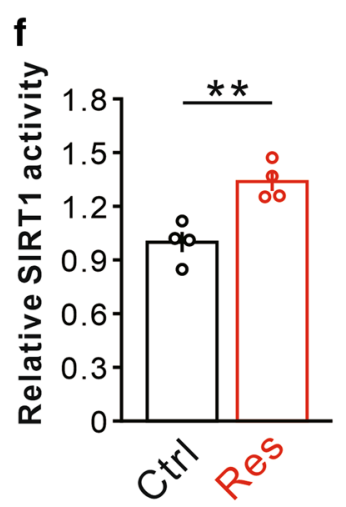

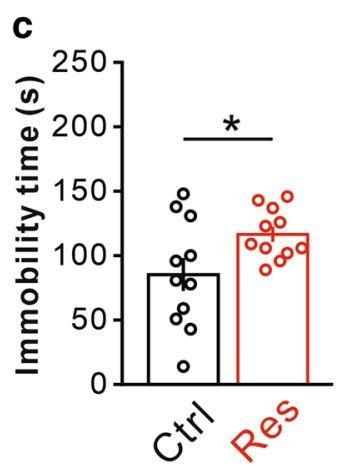

g

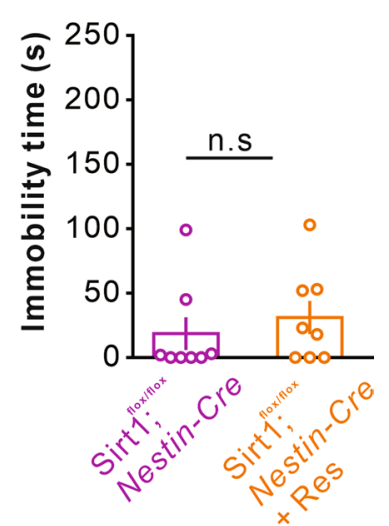

Fig. 1 The 24-h-restraint stress increased SIRT1 activity in hippocampus. a 24-h-restraint mice were subjected to acute restraint for $24 \mathrm{~h}$ and used for behavioral or biochemical experiments 5 weeks later. $\mathbf{b}$ Decreased the long-term depressive-like behaviors of SPT $\left(n=7\right.$ per group), ${ }^{*} p<0.05$. c Increased immobility time in restraint mice in the forced swimming test $\left(n=11\right.$ per group); ${ }^{*} p<0.05$. $\mathbf{d}$ No difference in levels of SIRT1 ( $n=4$ per group). e Levels of SIRT1 mRNA in the hippocampus ( $n=4$ per group). $\mathbf{f}$ Increased SIRT1 activity in restraint group than that in control group $\left(n=4\right.$ per group); ${ }^{* *} p<0.01$. g The duration of immobility time in forced swim test was no significant change in the conditional Sirt $1 \mathrm{KO}$ mice after 24-h-restraint stress ( $n=8$ per group). Data are presented as mean \pm SEM. Ctrl control, Res restraint, n.s. no significance 
tests: $p$ values $<0.05$ were considered statistically significant.

\section{Results}

The SIRT1 activity is increased in the 24-h-restraint depressive mice

Consistent with our previous report, the 24-h-restraint stress could produce long-term depressive-like phenotypes including deficits in sucrose preference test and forced swim test (Fig. 1a-c). To investigate the expression of SIRT1, we harvested hippocampal samples from 24-h-restraint (Res) and control (Con) mice 5 weeks after the modeling procedure. The qPCR and Western blot experiments were conducted. The protein and RNA levels of SIRT1 did not change in the hippocampus (Fig. 1d, e). However, SIRT1 activity was remarkably higher in the restraint mice than that in the control mice (Fig. 1f). As previous research reported that the brain-special conditional Sirt1 knockout mice displayed antidepressant behaviors [8], we subjected the mice to the 24-h-restraint

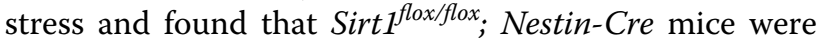
resistant to this stress in FST (Fig. 1g). These results suggested us that SIRT1 may play an important role in depression.

\section{Nicotinamide rescues depressive-like behaviors} without inhibiting SIRT1 but increasing ATP

Based on this finding, we chose NAM, an inhibitor of SIRT1, to investigate whether inhibiting SIRT1 activity could alleviate depressive phenotypes. Mice were treated with NAM for 5 weeks since 2 days after the 24-h restraint and conducted similar behavioral tests mentioned above. The experimental process is illustrated in a schematic in Fig. 2a. The sucrose preference of the restraint mice was significant enhanced after NAM treatment (Fig. 2b). The duration of immobility time in forced swim test was significantly declined in the restraint mice with NAM (Fig. 2c). NAM could not change the expression of SIRT1 or RNA (Fig. 2d, e). However, SIRT1 activity was further increased by NAM (Fig. 2f). Therefore, we hypothesized that NAM might play a role in rescue depression through other ways. It was reported that NAM was the precursor of $\mathrm{NAD}^{+}$, an essential co-enzyme of redox reactions for ATP production [12]. There were also evidences showing that the depression was associated with a decrease of ATP $[18,19]$. As our previous study found ATP was reduced in the 24-h-restraint depressive mice [9], we decided to detect the ATP level after NAM administration. Interestingly, we found that NAM could effectively reverse the ATP reduction caused by restraint stress in

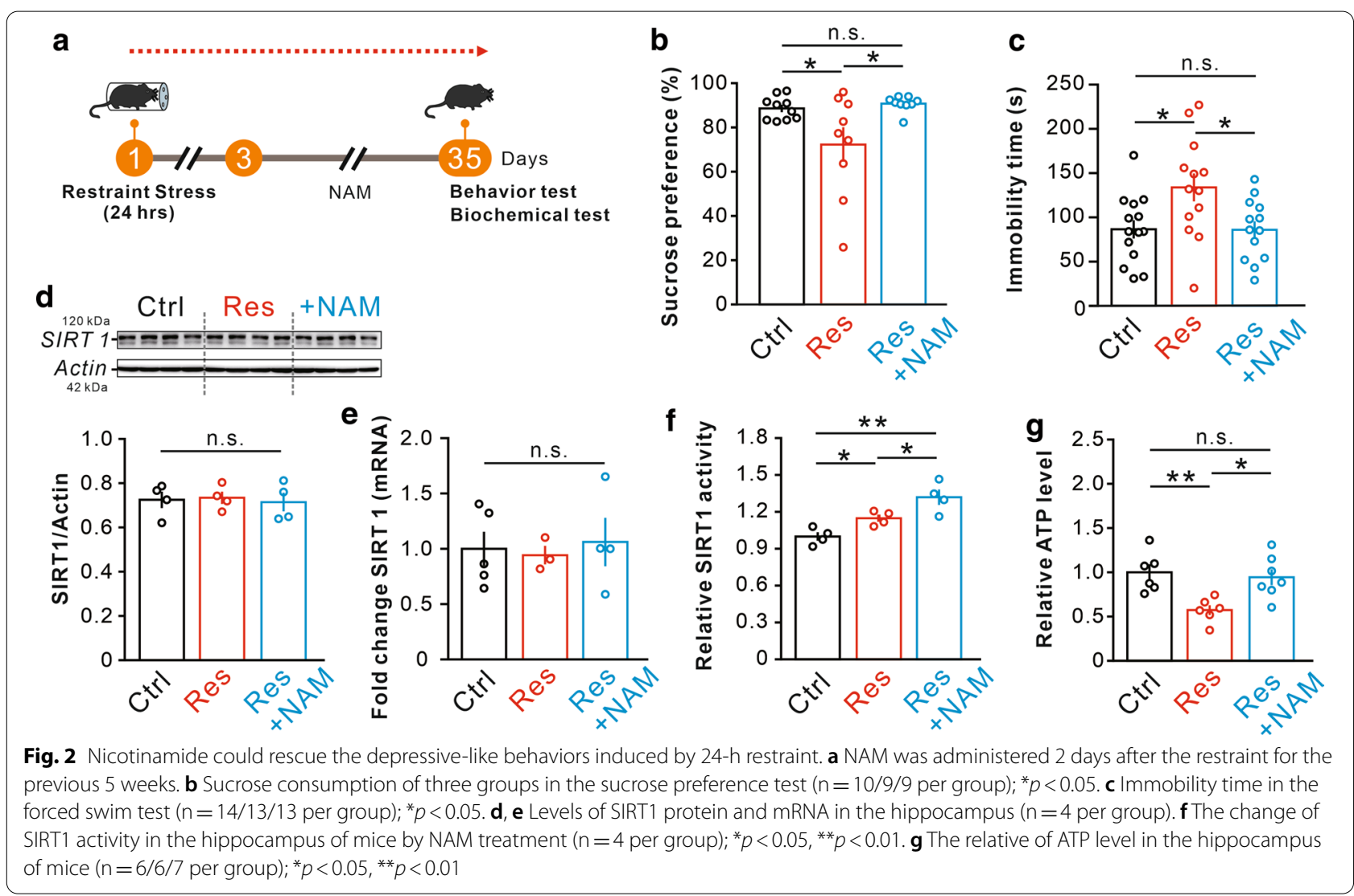


the hippocampus of the mice (Fig. 2g). These data suggested that ATP, instead of SIRT1 activity, plays a crucial role in regulating depression.

\section{The conditional Sirt 1 knockout mice exhibit antidepressant} behaviors and increased ATP levels

Because the Sirt $1^{\text {flox/flox }}$; Nestin-Cre exhibited antidepressant behaviors, we wondered if the phenotypes were related with ATP level when SIRT1 was absent. Western blot results showed efficient deletion of Sirt1 in mice (Fig. 3a). The Sirt flox/flox; Nestin-Cre mice exhibited reduced immobility times in the forced swimming test

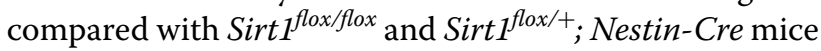
(Fig. 3b). Accordingly, the ATP level in the hippocampus of Sirt flox/flox; Nestin-Cre mice was significantly higher than that in Sirt $1^{f l o x f f l o x}$ and Sirt $1^{\text {flox/+ }}$; Nestin-Cre mice (Fig. 3c).

As a summary (Fig. 3d), restraint depressive mice shown the higher SIRT1 activity and lower ATP level in the hippocampus, NAM increased the ATP level and SIRT1 activity, attenuated depressive-like behaviors. Besides, the level of ATP was increased in Sirt $1^{\text {flox/flox; }}$; Nestin-Cre mice with antidepressant behaviors. The findings indicated that the ATP played a vital role in the regulation of depression independent of SIRT1.

\section{Discussion}

Clinical studies have demonstrated that NAM can stably improve the incidence of depression in patients, but the mechanism remains uncertain. NAM was previously thought to regulate a variety of physiological functions with the change of SIRT1. For example, Mitchell, S. J et al. found that chronic NAM supplementation could improve health span measures in mice without extending lifespans, and that enhanced acetylation of some SIRT1 targets in a diet and in NAM act in a dose-dependent manner [20]. However, Hwang et al. doubted the interpretation of results in studies that have used NAM as a SIRT1 inhibitor. They thought that NAM was an
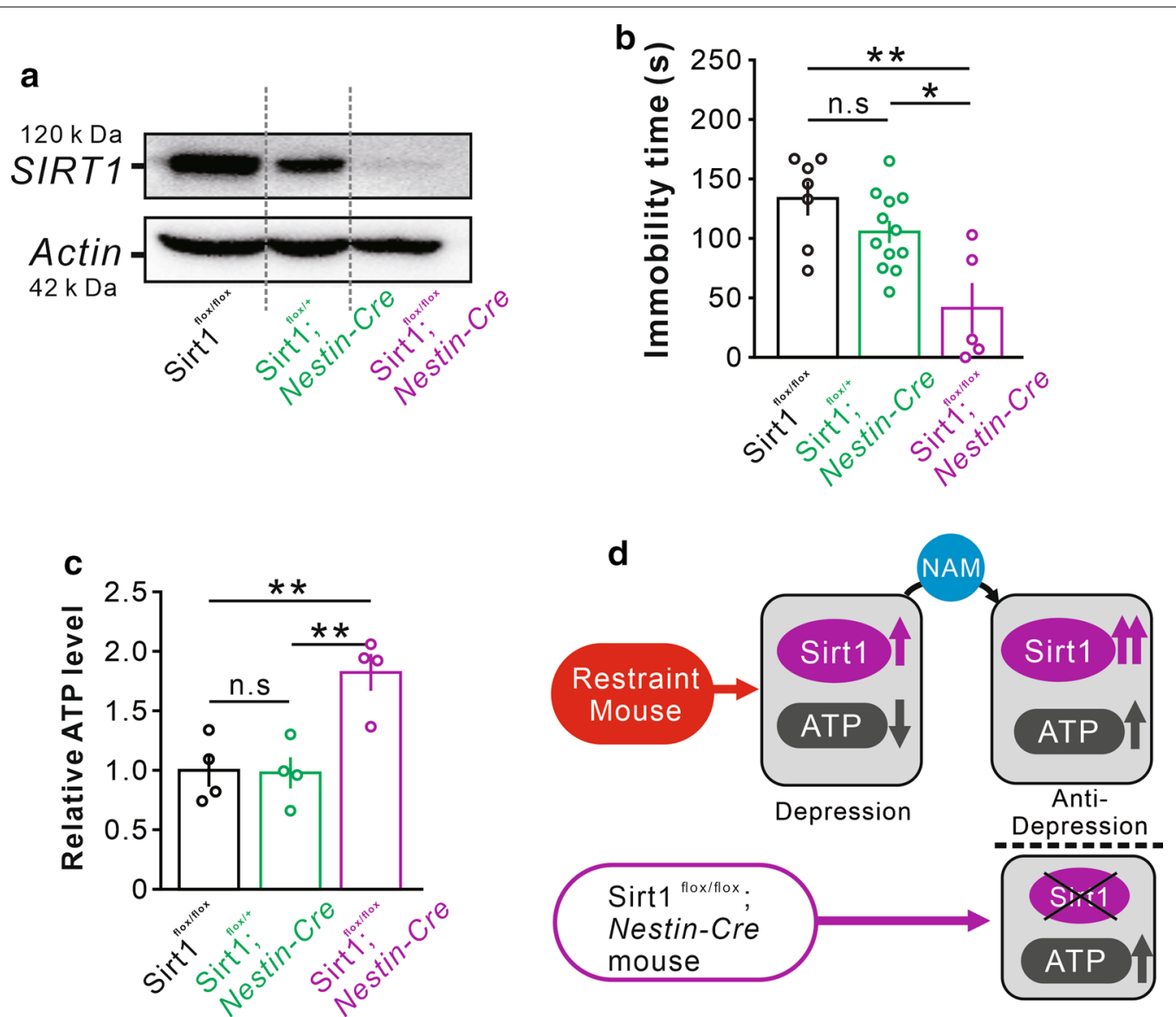

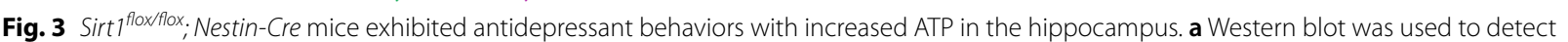
SIRT1 expression. $\mathbf{b}$ Immobility time in the forced swim test of different groups $\left(n=7 / 12 / 5\right.$ per group); ${ }^{*} p<0.05,{ }^{* *} p<0.01$. c The relative of ATP level in the hippocampus ( $n=4$ per group); ${ }^{* *} p<0.01$. d Schematic representation of key molecules in the restraint model and Sirt $7^{\text {flox }}$ flox; Nestin-Cre mice 
inhibitor of SIRT1 in vitro, while it could be a stimulator in cells [21]. Because SIRT1 activity was enhanced in the restraint depressive mice, we initially hypothesized that NAM could mediate depression by reducing SIRT1 activity. However, the application of NAM significantly reversed the depressive behaviors but increased SIRT1 activity further. These results showed that the change of SIRT1 activity was not consistent with the depressive phenotypes in mice. We also found that the Sirt $1^{\text {flox/ }}$ ${ }^{\text {flox }}$; Nestin-Cre mice exhibited antidepressant behaviors, while Sirt1 was deleted in brain. Combination with the discrepant changes of SIRT1 in depression, we speculated that SIRT1 could not play a direct role in the pathogenesis of depression.

Decreased ATP metabolism has been reported in patients with MDD and in animal models of depression $[19,22,23]$. Importantly, we found the level of ATP was reduced in the restraint model for depression, consistent with previous study [9], and recovered by the administration of NAM. As NAM could increase $\mathrm{NAD}^{+}$levels that modulate the mitochondrial production of ATP through oxidative phosphorylation [12]. We concluded that NAM reduced depression-like behavior by increasing the ATP level in our mouse model. Besides, the further increased SIRT1 activity may also due to the raised $\mathrm{NAD}^{+}$levels after NAM administration [21, 24, 25]. We also found that Sirt1 floxfllox; Nestin-Cre mice were anti-depression with higher ATP level in the hippocampus of brain. However, the level of ATP in Sirt flox/flox; Nestin-Cre mice resilient to 24-h-restraint stress need to be detected in the future. According to these results, we speculated that the level of ATP could regulate depressive-like behaviors, whether the SIRT1 activity was increased or deleted.

These results remind us that the role of the SIRT1mediated pathogenesis of depression in the model of environmental stress must be reconsidered. This role may resolve some of the controversies surrounding the change of SIRT1 in depression. Our study also provides new insights into the use of NAM in treating depression.

\begin{abstract}
Abbreviations
NAM: Nicotinamide; SIRT1: Sirtuin 1; ATP: Adenosine triphosphate; MDD: Major depression disorder; $\mathrm{NAD}^{+}$: Nicotinamide adenine dinucleotide; CAC: Citric acid cycle; FST: Force swim test; SPT: Sucrose preference test; DG: Dentate gyrus; NAc: Nucleus accumbens; BSA: Bovine serum albumin; PVDF: Polyvinylidene fluoride; RT-PCR: Real-time polymerase chain reaction; SEM: Standard error of the mean; ANOVA: Analysis of variance.
\end{abstract}

\section{Acknowledgements}

The authors are grateful to all those who participated in this research for their contributions.

\section{Authors' contributions}

WL conceived, designed, and planned the project as well as reviewed the statistical analyses and wrote the manuscript. ZL conducted the experiments, completed the statistical analyses, and wrote the manuscript. $C L, X F, Y K, X Z$,
LC, JS, YZ, ET and GH helped in conducting the experiments. All authors read and approved the final manuscript.

\section{Funding}

This study is supported by Shanghai Education Commission Research and Innovation Program (2019-01-07-00-02-E00037), National Key Research and Development Program of China (2018YFE0126700), Program of Shanghai Subject Chief Scientist (17XD1401700), "1 11"Program of Higher Education Discipline Innovation, and Shanghai Jiao Tong University Scientific and Technological Innovation Funds.

\section{Availability of data and materials}

All data generated or analysed during this study are included in this published article.

\section{Ethics approval and consent to participate}

All animal experiments were approved by the Institutional Animal Care and Use Committee of Shanghai Jiao Tong University.

\section{Consent for publication}

Not applicable.

\section{Competing interests}

The authors declare that they have no competing interests.

Received: 17 September 2020 Accepted: 17 November 2020

Published online: 23 November 2020

References

1. Asokan GV, et al. The magnitude and correlates of geriatric depression using Geriatric Depression Scale (GDS-15) - a Bahrain perspective for the WHO 2017 campaign "Depression - let's talk." Perspect Public Health. 2019;139(2):79-87.

2. Consortium C. Sparse whole-genome sequencing identifies two loci for major depressive disorder. Nature. 2015;523(7562):588-91.

3. Lei Y, et al. SIRT1 in forebrain excitatory neurons produces sexually dimorphic effects on depression-related behaviors and modulates neuronal excitability and synaptic transmission in the medial prefrontal cortex. Mol Psychiatry. 2020;25(5):1094-111.

4. Lu G, et al. Role and possible mechanisms of Sirt1 in depression. Oxid Med Cell Longev. 2018;2018:8596903.

5. Abe-Higuchi $\mathrm{N}$, et al. Hippocampal sirtuin 1 signaling mediates depression-like behavior. Biol Psychiatry. 2016;80(11):815-26.

6. Ferland $\mathrm{CL}$, et al. Sirtuin activity in dentate gyrus contributes to chronic stress-induced behavior and extracellular signal-regulated protein kinases 1 and 2 cascade changes in the hippocampus. Biol Psychiatry. 2013;74(12):927-35.

7. Kim HD, et al. SIRT1 mediates depression-like behaviors in the nucleus accumbens. J Neurosci. 2016;36(32):8441-52.

8. Libert S, et al. SIRT1 activates MAO-A in the brain to mediate anxiety and exploratory drive. Cell. 2011;147(7):1459-72.

9. Chu $X$, et al. 24-hour-restraint stress induces long-term depressive-like phenotypes in mice. Sci Rep. 2016;6:32935.

10. Prousky JE. Vitamin B3 for depression: case report and review of the literature. J Orthomol Med. 2010:25(3):137-47.

11. Jonsson BH. Nicotinic acid long-term effectiveness in a patient with bipolar type ii disorder: a case of vitamin dependency. Nutrients. 2018;10(2):134.

12. Song SB, et al. Diverse therapeutic efficacies and more diverse mechanisms of nicotinamide. Metabolomics. 2019;15(10):137.

13. Bitterman $\mathrm{KJ}$, et al. Inhibition of silencing and accelerated aging by nicotinamide, a putative negative regulator of yeast sir2 and human SIRT1. J Biol Chem. 2002;277(47):45099-107.

14. Avalos JL, Bever KM, Wolberger C. Mechanism of sirtuin inhibition by nicotinamide: altering the $\mathrm{NAD}(+)$ cosubstrate specificity of a Sir2 enzyme. Mol Cell. 2005;17(6):855-68. 
15. Green $\mathrm{KN}$, et al. Nicotinamide restores cognition in Alzheimer's disease transgenic mice via a mechanism involving sirtuin inhibition and selective reduction of Thr231-phosphotau. J Neurosci. 2008;28(45):11500-10.

16. Williams PA, et al. Vitamin B3 modulates mitochondrial vulnerability and prevents glaucoma in aged mice. Science. 2017;355(6326):756-60.

17. Song YM, et al. Metformin alleviates hepatosteatosis by restoring SIRT1mediated autophagy induction via an AMP-activated protein kinaseindependent pathway. Autophagy. 2015;11(1):46-59.

18. Bansal Y, Kuhad A. Mitochondrial dysfunction in depression. Curr Neuropharmacol. 2016;14(6):610-8.

19. Cao X, et al. Astrocyte-derived ATP modulates depressive-like behaviors Nat Med. 2013;19(6):773-7.

20. Mitchell SJ, et al. Nicotinamide improves aspects of healthspan, but not lifespan, in mice. Cell Metab. 2018;27(3):667-676 e4.

21. Hwang ES, Song SB. Nicotinamide is an inhibitor of SIRT1 in vitro, but can be a stimulator in cells. Cell Mol Life Sci. 2017;74(18):3347-62.

22. Gardner A, et al. Alterations of mitochondrial function and correlations with personality traits in selected major depressive disorder patients. J Affect Disord. 2003;76(1-3):55-68.
23. Xie $X$ et al Nicotinamide mononucleotide ameliorates the depressionlike behaviors and is associated with attenuating the disruption of mitochondrial bioenergetics in depressed mice. J Affect Disord. 2020;263:166-74

24. Chandrasekaran $\mathrm{K}$, et al. Role of mitochondria in diabetic peripheral neuropathy: influencing the NAD(+)-dependent SIRT1-PGC-1alpha-TFAM pathway. Int Rev Neurobiol. 2019;145:177-209.

25. Choi SE, Kemper JK. Regulation of SIRT1 by microRNAs. Mol Cells. 2013;36(5):385-92.

\section{Publisher's Note}

Springer Nature remains neutral with regard to jurisdictional claims in published maps and institutional affiliations.
Ready to submit your research? Choose BMC and benefit from:

- fast, convenient online submission

- thorough peer review by experienced researchers in your field

- rapid publication on acceptance

- support for research data, including large and complex data types

- gold Open Access which fosters wider collaboration and increased citations

- maximum visibility for your research: over $100 \mathrm{M}$ website views per year

At BMC, research is always in progress.

Learn more biomedcentral.com/submissions 